\section{To: (Receiving Organtzation) \\ Distribution}

5. Proj./Prog/Dept./Dlv.:

Radiological Engineering

8. Originator Remarks:

"Dose Rate Calculations for Rotary Mode Core Sampling Exhauster" is being issued as RPP-6549.

11. Recelver Remarks:

11A. Design Baseline Document? $\square$ Yes $\bigotimes$ No

3. From: (Originating Organization)
TFC Radiological Control
6. Dealgn Authority/Deaign Agent/Cog. Engr.:
D. J. Foust

3. From: (Originating Organization)

D. J. Foust

\section{Related EDT No.: \\ N/A \\ 7. Purchase Order No. \\ N/A \\ 9. Equip./Component No.: \\ N/A}

10. Syatem/Bidg./Faclity:

N/A

12. Major Assm. Dwg. No.:

N/A

13. Permit/Permit Application No.:

N/A

14. Requifed Response Date:

\section{$\mathrm{N} / \mathrm{A}$}

\begin{tabular}{|l|l|}
\hline \multicolumn{2}{|l|}{15.} \\
\hline $\begin{array}{l}\text { (A) } \\
\text { Ifem } \\
\text { No. }\end{array}$ & (B) Document/Drawing No. \\
\hline 1 & RPP-6549 \\
\hline & \\
\hline & \\
\hline & \\
\hline & \\
\hline & \\
\hline & \\
\hline
\end{tabular}

DATA TRANSMITTED

\begin{tabular}{l|l|l}
$\begin{array}{l}\text { (C) Sheet } \\
\text { No. }\end{array}$ & (D) Rev. \\
No. & (E) Title or Description of Data Transmitted
\end{tabular} Dose Rate Calculations for

\begin{tabular}{l|l|l} 
N/A & 0 & Dose Rate Calculations for \\
\hline
\end{tabular}
Rotary Mode Core Sampling Exhauster

16.

KEY

\begin{tabular}{c|l}
\hline Approval Designator (F) & \\
\hline E, S, O, D OR N/A & 1. Approval \\
(See WHC.CM-3-5, & 2. Release \\
Sec. 12.7) & 3. Information \\
\hline
\end{tabular}

17.

Reason for Tranemittal (G)

4. Review

5. Post-Review

6. Dist. (Receipt Acknow. Required)

SIGNATUREIOISTRIBUTION

(See Approval Designtor for required signatures)

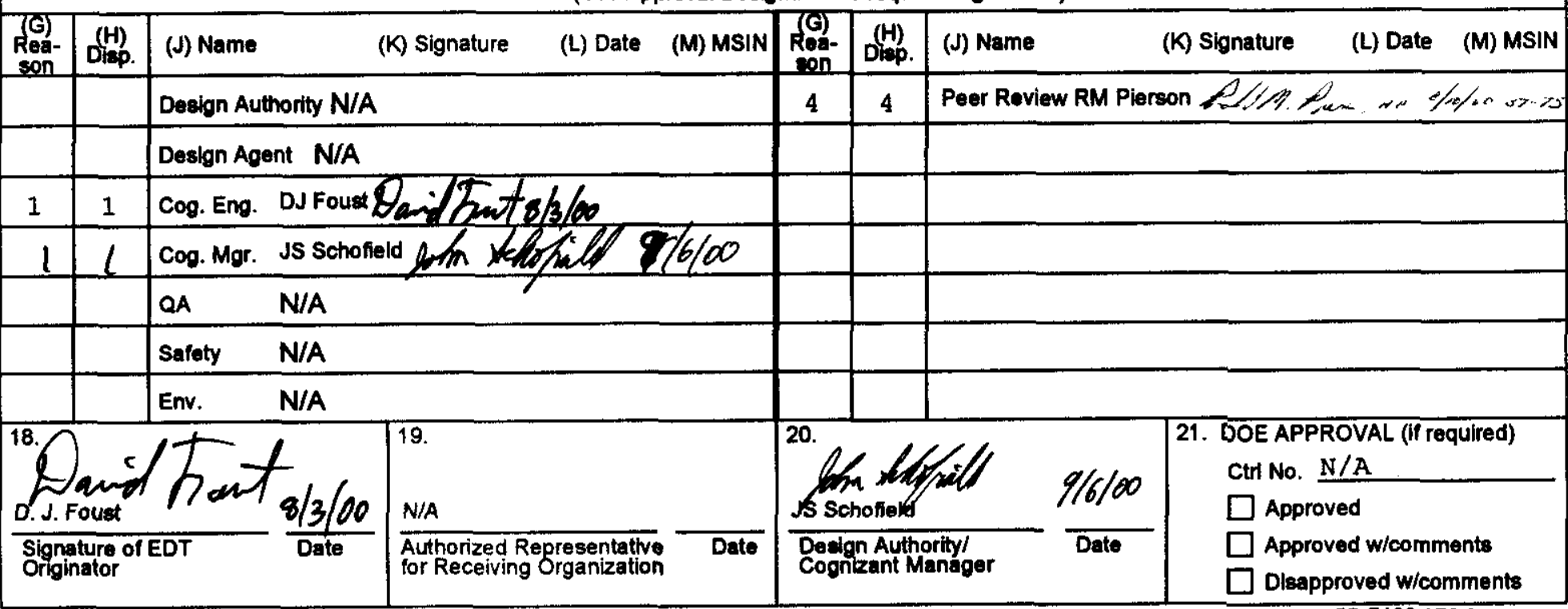

Disposition (H) \& (I)

\section{Approved \\ 4. Reviewed no/comment \\ 5. Reviewed w/comment}

6. Receipt acknowledged
(F)

(G)

$(\mathrm{H})$

Origi- Recelv-

Approval Reason nator or

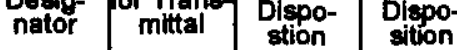

\begin{tabular}{|l|l|l|l|}
\hline N/A & 3 & 1 & \\
\hline
\end{tabular}




\section{DISTRIBUTION SHEET}

\begin{tabular}{l|l|}
\hline $\begin{array}{l}\text { To } \\
\text { Distribution }\end{array}$ & $\begin{array}{l}\text { From } \\
\text { D. J. Foust }\end{array}$ \\
\hline Project TitleWork Order & \\
RPP-6549 - Dose Rate Calculations for Rotary Mode Core Sampling Exhauster
\end{tabular}

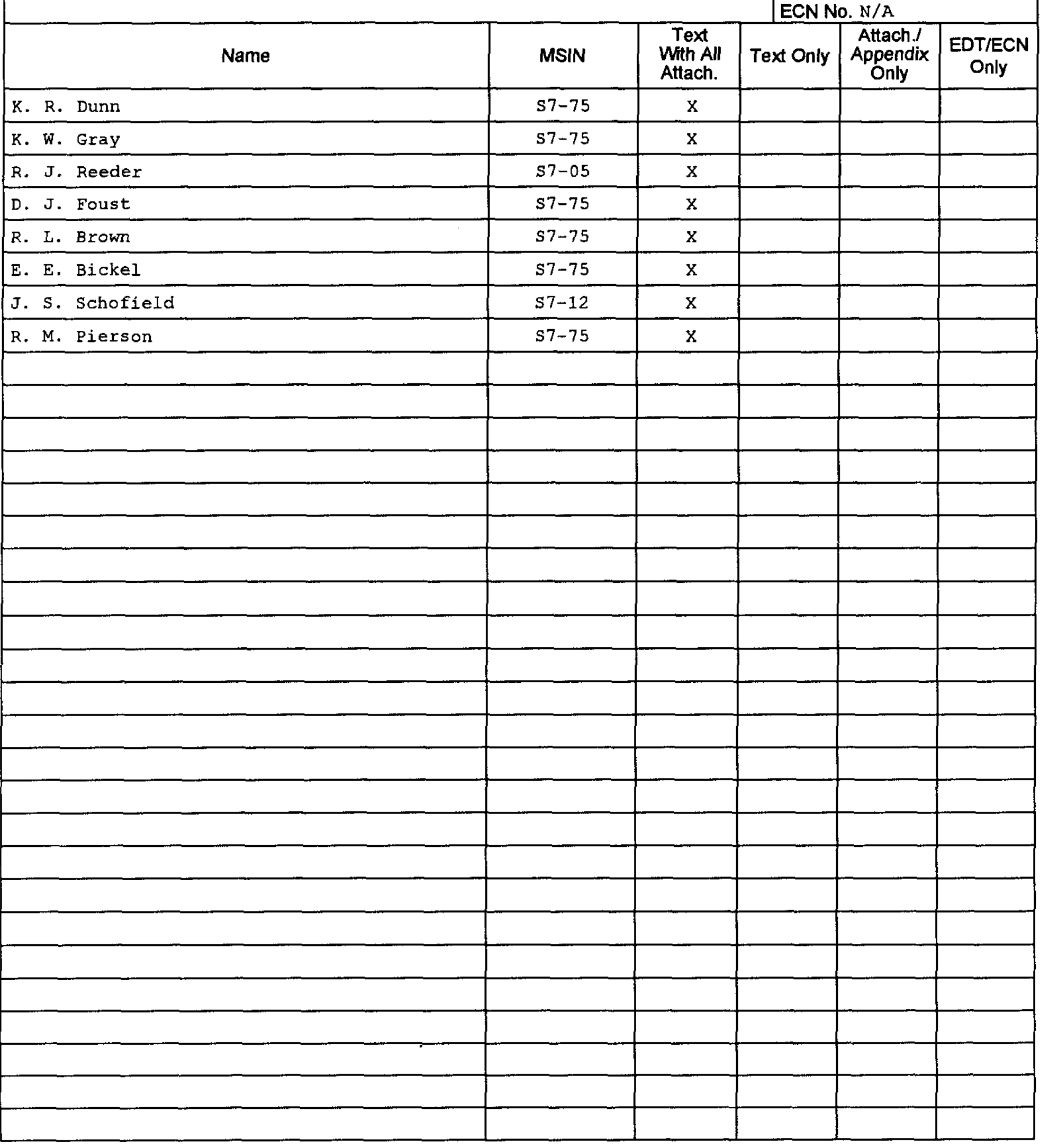




\title{
Dose Rate Calculations for Rotary Mode Core Sampling Exhauster
}

\author{
D. J. Foust \\ CH2M Hill Hanford Group Inc. \\ Richland, WA 99352 \\ U.S. Department of Energy Contract DE-AC06-99RL14047

$\begin{array}{lll}\text { EDT/ECN: } & \text { EDT }-630276 & \text { UC: } 2080 \\ \text { Cost Center: } & 79700 & \text { Charge Code: } 102266 \\ \text { B\&R Code: } & \text { N/A } & \text { Total Pages: } 22\end{array}$

Key Words

RMCST; HEPA; exhauster; core sampling

Abstract:

This document provides calculated estimated dose rates for three external locations on the rotary mode core sampling exhauster HEPA filter housing.

TRADEMARK DISCLAIMER. Reference herein to any specific commercial product, process, or service by trade name, trademark, manufacturer, or otherwise, does not necessarily constitute or imply its endorsement, recommendation, or favoring by the United States Government or any agency thereof or its contractors or subcontractors.

Printed in the United States of America. To obtain copies of this document, contact: Document Control Services, P.O. Box 950, Mailstop H6-08, Richland WA 99352, Phone (509) 372-2420; Fax (509) 376-4989.
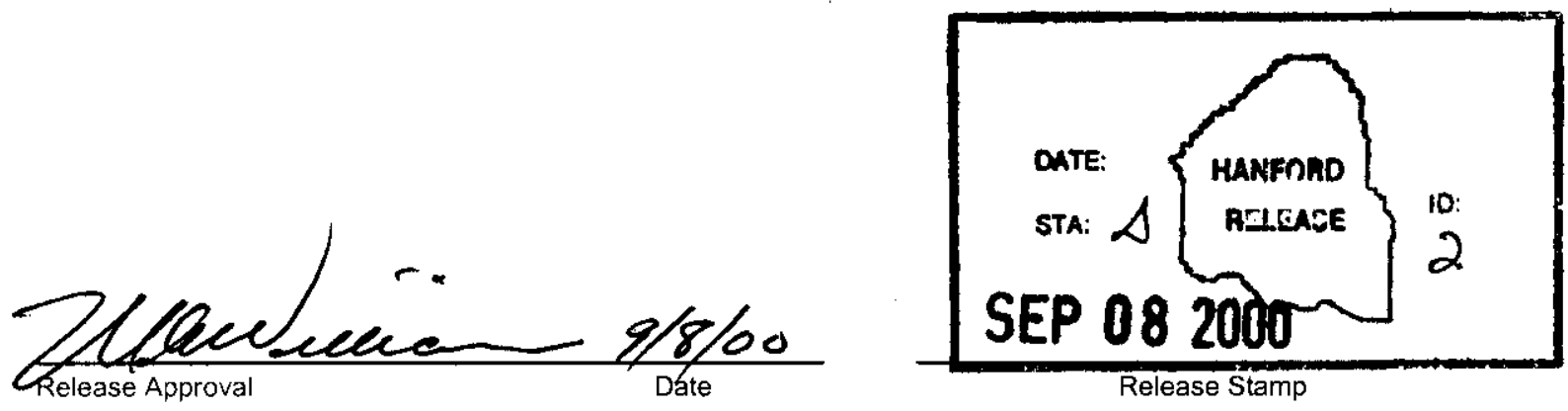

\section{Approved For Public Release}




\section{Purpose}

This document provides the calculated estimated dose rates for three extemal locations on the Rotary Mode Core Sampling (RMCS) exhauster HEPA filter housing, per the request of Characterization Field Engineering.

\section{Calculations}

Dose rates for nine cases, as described in table 2, were calculated utilizing the Microshield ${ }^{2}$ computer-modeling program. The geometry utilized is shown in figures 1 and 2 below. The isotopic concentration utilized in the source term is shown in table 1.

Because of limitations in the Microshield computer-model, it was not possible the jingodel the filters as rectangular solids in cases 2, 3, 4, 6, 7 and 8. For these cases, it was necessary to model the filters as rectangular plane sources, and thus, the results for these cases are estimates only, and may not be directly comparable to the results for cases 1,5 and 9 . Because of the lack of self shielding in the planar model, the calculated dose rates for these cases is likely to be higher than actual measurements.

\section{References}

- Interoffice Memo \# 74910-00-010, “Dose Rate Calculations for Rotary Mode Core Sampling Exhauster, June 6, 2000

\begin{tabular}{|c|c|c|}
\hline \multirow{3}{*}{$\begin{array}{c}\text { Table 1 } \\
\text { SST Solids Radionuclide Concentrations } \\
\text { To Use for RMCs Exhauster Housing } \\
\text { Dose Rate Calculations }\end{array}$} \\
\hline \multirow{2}{*}{ Isotope } & \multicolumn{2}{|c|}{ Concentration } \\
\cline { 2 - 3 } & $\mathrm{Bq} / \mathrm{L}$ & $\mathrm{Cl} / \mathrm{L}$ \\
\hline${ }^{60} \mathrm{Co}$ & $4.2 \times 10^{8}$ & 0.011 \\
\hline${ }^{90} \mathrm{Sr}$ & $1.6 \times 10^{12}$ & 43.2 \\
\hline${ }^{90} \mathrm{Y}$ & $1.6 \times 10^{12}$ & 43.2 \\
\hline${ }^{137} \mathrm{Cs}$ & $1.0 \times 10^{11}$ & 2.7 \\
\hline${ }^{137 \pi} \mathrm{Ba}$ & $9.5 \times 10^{10}$ & 2.6 \\
\hline${ }^{154} \mathrm{Eu}$ & $5.8 \times 10^{9}$ & 0.16 \\
\hline
\end{tabular}

\footnotetext{
'Interoffice Memo * 74910-00-010, "Dose Rate Calculations for Rotary Mode Core Sampling Exhauster, June 6, 2000

${ }^{2}$ MicroShield is a registered trademark of Grove Engineering Inc.
} 


\begin{tabular}{|c|c|c|}
\hline \multicolumn{3}{|c|}{ Table 2} \\
\hline $\begin{array}{l}\text { Case } \\
\text { Number }\end{array}$ & Description & $\begin{array}{c}\text { Dose Rate } \\
(\mathrm{mR} / \mathrm{hr})\end{array}$ \\
\hline 1 & $\begin{array}{l}\text { Dose rate at point } \mathrm{A} \text { with } 1 \mathrm{~L} \text { of waste distributed } \\
\text { evenly throughout the pre-filter. }\end{array}$ & 5,129 \\
\hline 2 & $\begin{array}{l}\text { Dose rate at point } \mathrm{B} \text { with } 1 \mathrm{~L} \text { of waste distributed } \\
\text { evenly throughout the pre-filter. }\end{array}$ & $729^{1}$ \\
\hline 3 & $\begin{array}{l}\text { Dose rate at point } C \text { with } 1 \mathrm{~L} \text { of waste distributed } \\
\text { evenly throughout the pre-filter. }\end{array}$ & $245^{1}$ \\
\hline 4 & $\begin{array}{l}\text { Dose rate at point } A \text { with } 1 L \text { of waste distributed } \\
\text { evenly throughout the } 1^{\text {th }} \text { HEPA. }\end{array}$ & $729^{1}$ \\
\hline 5 & $\begin{array}{l}\text { Dose rate at point } B \text { with } 1 \mathrm{~L} \text { of waste distributed } \\
\text { evenly throughout the } 1^{\text {st }} \text { HEPA. }\end{array}$ & 5,085 \\
\hline 6 & $\begin{array}{l}\text { Dose rate at point } C \text { with } 1 \mathrm{~L} \text { of waste distributed } \\
\text { evenly throughout the } 1^{\text {st }} \mathrm{HEPA} \text {. }\end{array}$ & $364^{1}$ \\
\hline 7 & $\begin{array}{l}\text { Dose rate at point } A \text { with } 1 \text { L of waste distributed } \\
\text { evenly throughout the } 2^{\text {nd }} \text { HEPA. }\end{array}$ & $245^{1}$ \\
\hline 8 & $\begin{array}{l}\text { Dose rate at point } B \text { with } 1 \mathrm{~L} \text { of waste distributed } \\
\text { evenly throughout the } 2^{\text {nd }} \text { HEPA. }\end{array}$ & $364^{1}$ \\
\hline 9 & $\begin{array}{l}\text { Dose rate at point } C \text { with } 1 \mathrm{~L} \text { of waste distributed } \\
\text { evenly throughout the } 2^{\text {nd }} \text { HEPA. }\end{array}$ & 5,085 \\
\hline
\end{tabular}

\begin{tabular}{|c|c|c|c|c|c|}
\hline \multicolumn{6}{|c|}{$\begin{array}{c}\text { Table } 3 \\
\text { Shield Materials }\end{array}$} \\
\hline Component & $\begin{array}{c}\text { Dimension } \\
\text { (inches) }\end{array}$ & Material & $\begin{array}{l}\text { Density } \\
\left(\mathrm{gm} / \mathrm{cm}^{3}\right)\end{array}$ & $\begin{array}{l}\text { Elemental } \\
\text { Component }\end{array}$ & $\begin{array}{l}\% \text { by } \\
\text { Weight }\end{array}$ \\
\hline \multirow{3}{*}{$\begin{array}{l}\text { HEPA } \\
\text { Filter }\end{array}$} & \multirow{3}{*}{11.2} & $\mathrm{Al}$ & 0.03 & $\mathrm{Al}$ & 100 \\
\hline & & \multirow{2}{*}{$\mathrm{SiO}_{2}$} & \multirow{2}{*}{0.02} & Si & 47 \\
\hline & & & & 0 & 53 \\
\hline $\begin{array}{l}\text { HEPA } \\
\text { Frame }\end{array}$ & 0.075 & $\mathrm{Fe}$ & 7.86 & $\mathrm{Fe}$ & 100 \\
\hline $\begin{array}{l}\text { Air } \\
\text { Gap }\end{array}$ & 4.25 & & 0.00122 & & 100 \\
\hline $\begin{array}{l}\text { Duct } \\
\text { Wall }\end{array}$ & 0.25 & $\mathrm{Fe}$ & 7.86 & $\mathrm{Fe}$ & 100 \\
\hline $\begin{array}{c}\text { Foam } \\
\text { Insulation }\end{array}$ & 1.13 & C & 0.20 & C & 100 \\
\hline $\begin{array}{l}\text { 'Interofilice Mer } \\
\text { Sampling Exha }\end{array}$ & $\begin{array}{l}4910-00-0 \\
\text { June } 6,20 \\
\end{array}$ & 5080 & & Mode & \\
\hline
\end{tabular}


Dose Rate Calculations

Figure 1:

\section{REME Exhauster Housing Cross Section}

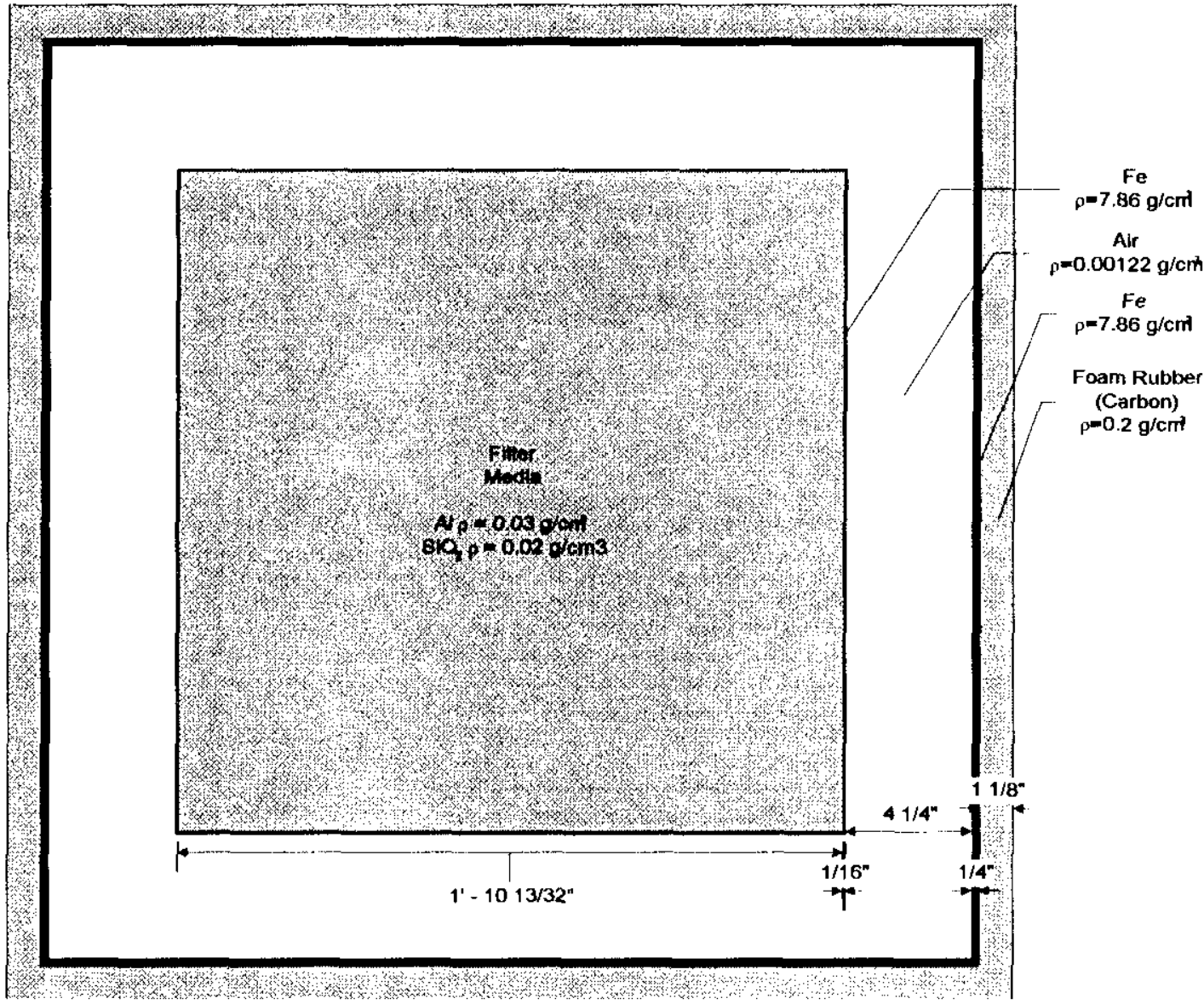

Figure 2:

RMCS Exhauster Housing

(External View Showing Dose Rate Locations for Calculations)

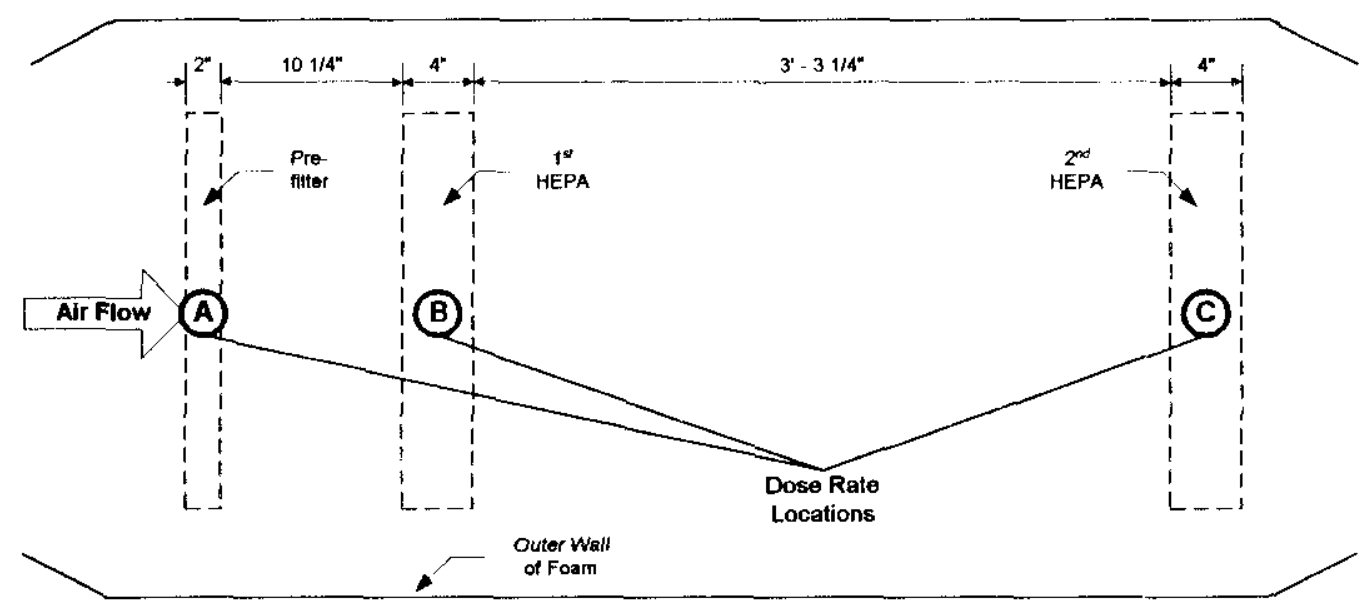


Page : 1

DOS File: RMCSEXH1MS5

Run Date: June 8, 2000

Run Time: 8:36:30 AM

Duration : 00:00:06
RPP-6549

Revision 0
River Protection Project - Radiological Control

Page 4 of 21

File Ref:

Date: $8 / 2 / 2000$

By: Eन

Checked:
Case Title: RMCSExh1

Description: RMCS Exhauster - Case 1

Geometry: 13 - Rectangular Volume

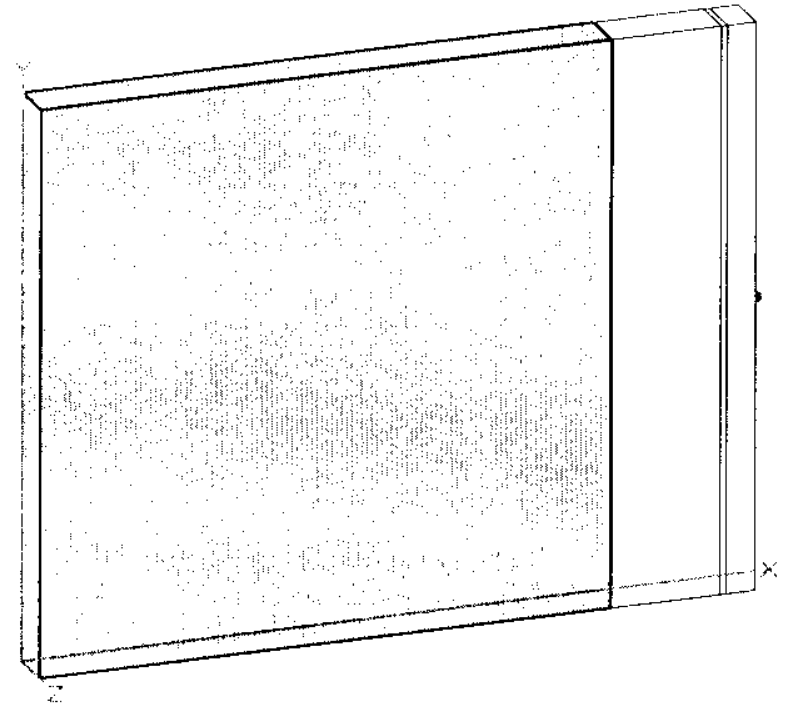

\begin{tabular}{lc} 
Nuclide & \multicolumn{1}{c}{ curies } \\
Ba-137m & $2.6000 e+000$ \\
Co-60 & $1.1000 e-002$ \\
Cs -137 & $2.7000 e+000$ \\
Eu-154 & $1.6000 e-001$ \\
Sr-90 & $4.3200 e+001$ \\
Y-90 & $4.3200 e+001$
\end{tabular}

Energy

MeV

0.03

0.04

0.05

0.1
Activity

photons/sec

$5.666 e+09$

$2.542 e+09$

$3.060 e+08$

$2.395 e+09$
The material reference is : Shield 3

$X$ Direction

$y$ Direction

Z Direction
Source Input

Grouping Method : Standard Indices

Number of Groups : 25

Lower Energy Cutoff : 0.015

Photons < 0.015 : Excluded

Library : Grove

$9.6200 e+010$

Source Dimensions

$56.896 \mathrm{~cm}$

$56.896 \mathrm{~cm}$

$\underline{x}$

$72.39 \mathrm{~cm}$

$2 \mathrm{ft} 4.5 \mathrm{in}$

Shield Name

Source

Shield 1

Shield 2

Shield 4

Air Gap becquerels

$4.0700 e+008$

$9.9900 e+010$

$5.9200 e+009$

$1.5984 e+012$

$1.5984 e+012$

Buildup

Integration Parameters

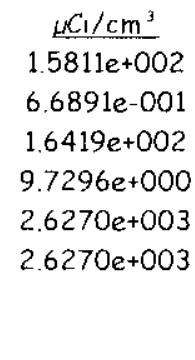

10

20

20 $\underline{z}$

$2.54 \mathrm{~cm}$

1.0 in

Shields

\begin{tabular}{rcl}
\multicolumn{1}{c}{ Dimension } & Material & Density \\
\cline { 2 - 3 } $1.64 \mathrm{e}+04 \mathrm{~cm}^{3}$ & HEPA & 0.05 \\
$.191 \mathrm{~cm}$ & Iron & 7.86 \\
$10.795 \mathrm{~cm}$ & Air & 0.00122 \\
$.635 \mathrm{~cm}$ & Iron & 7.86 \\
$2.87 \mathrm{~cm}$ & Carbon & 0.29 \\
& Air & 0.00122
\end{tabular}

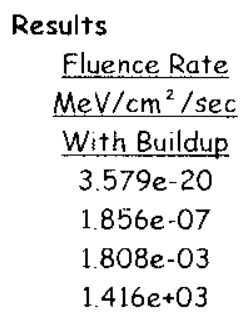

$5.08 \mathrm{~cm}$

Dose Points
$1 \mathrm{ft} 10.4 \mathrm{in}$

$2.0 \mathrm{in}$

$1 \mathrm{ft} 10.4$ in
$\frac{\mathrm{Bq} / \mathrm{cm}^{3}}{5.8499 e+006}$
$2.4750 e+004$
$6.0749 e+006$
$3.5999 e+005$
$9.7198 e+007$
$9.7198 e+007$

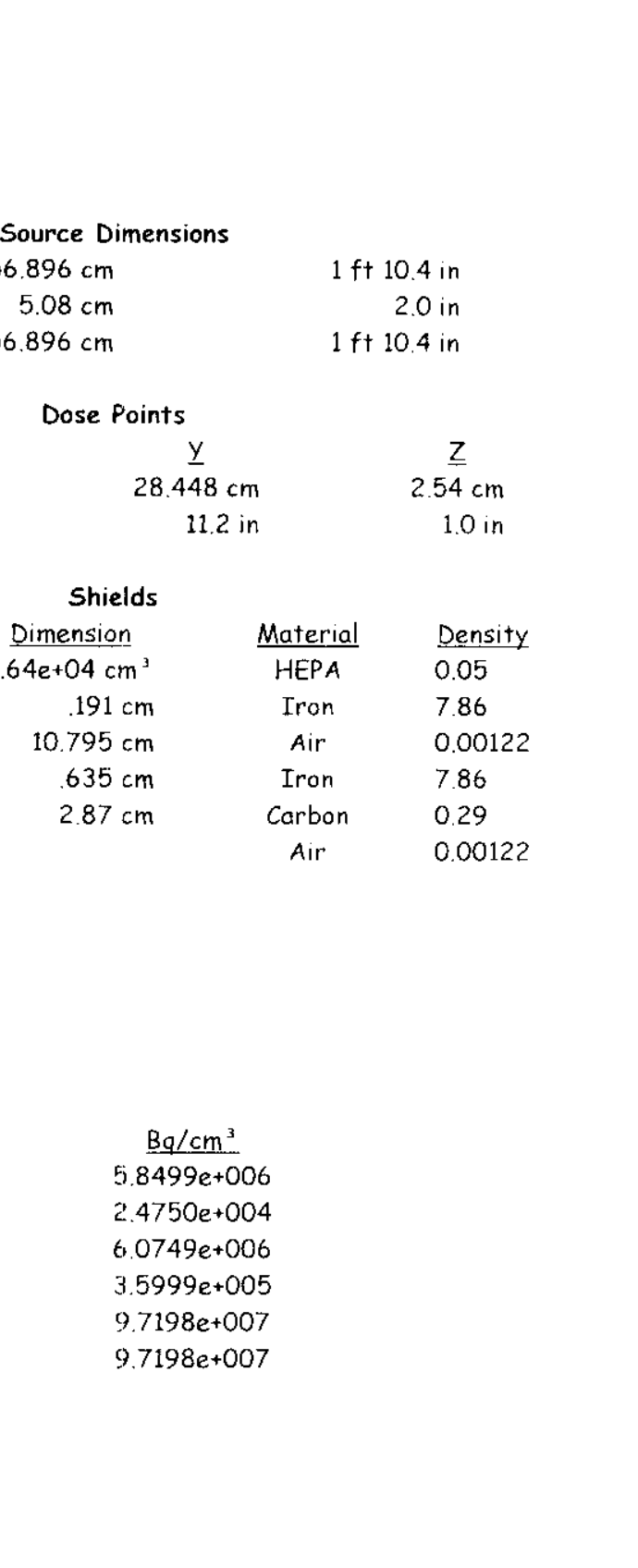


Page : 2

DOS File: RMCSEXH1.MS5

Run Date: June 8, 2000

Run Time: 8:36:30 AM

Duration: 00:00:06

\begin{tabular}{|c|c|c|c|c|c|}
\hline$\frac{\text { Energy }}{\underline{\text { MeV }}}$ & $\frac{\text { Activity }}{\text { photons/sec }}$ & $\begin{array}{l}\frac{\text { Fluence Rate }}{\text { MeV/cm } / \mathrm{cmec}^{2} / \mathrm{sec}} \\
\text { No Buildup }\end{array}$ & $\begin{array}{l}\frac{\text { Fluence Rate }}{\text { MeV/cm } / \mathrm{cm}^{2} / \mathrm{sec}} \\
\underline{\text { With Buildup }}\end{array}$ & $\begin{array}{c}\text { Exposure Rate } \\
\text { No Buildup } \\
\text { No }\end{array}$ & $\begin{array}{c}\frac{\text { Exposyre Rate }}{m \mathrm{~m} / \mathrm{hr}} \\
\text { With Buildup }\end{array}$ \\
\hline 0.2 & $4.043 e+08$ & $1.265 e+03$ & $2.569 e+03$ & $2.233 e+00$ & $4.534 e+00$ \\
\hline 0.4 & $4.224 e+07$ & $3.889 e+02$ & $7.162 e+02$ & $7.578 e-01$ & $1.395 e+00$ \\
\hline 0.5 & $1.282 e+07$ & $1.601 e+02$ & $2.781 e+02$ & $3.143 e-01$ & $5.458 e-01$ \\
\hline 0.6 & $8.704 e+10$ & $1.387 e+06$ & $2.290 e+06$ & $2.707 e+03$ & $4.469 e+03$ \\
\hline 0.8 & $2.309 e+09$ & $5.357 e+04$ & $8.196 e+04$ & $1.019 e+02$ & $1.559 e+02$ \\
\hline 1.0 & $2.228 e+09$ & $6.885 e+04$ & $9.988 e+04$ & $1.269 e+02$ & $1.841 e+02$ \\
\hline 1.5 & $2.717 e+09$ & $1.394 e+05$ & $1.851 e+05$ & $2.345 e+02$ & $3.113 e+02$ \\
\hline TOTALS: & $1.057 e+11$ & $1.651 e+06$ & $2.662 e+06$ & $3.174 e+03$ & $5.129 e+03$ \\
\hline
\end{tabular}


Page : 1

DOS File: RMDSEXH2.MS5

Run Date: August 3, 2000

Run Time: 8:08:32 AM

Duration : 00:00:01

Case Title: RCMSExh2

Description: RCMS Exhouster - Case 2

Geometry: 5 - Rectangular Area - Horizontal
Page 6 of 21

File Ref:

Date: $8 / 2 / 2000$

By

Checked

$0 \%$

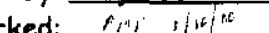

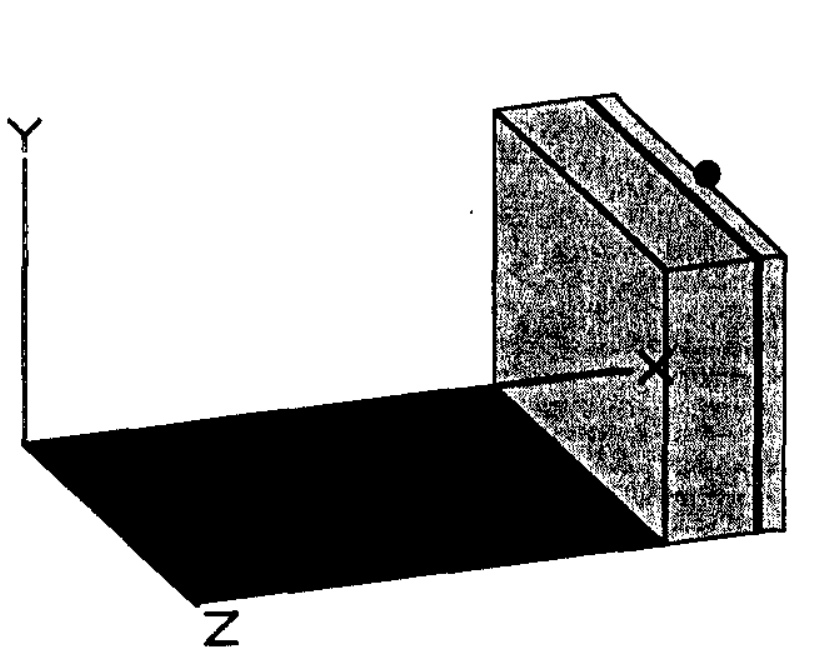

\begin{tabular}{lc} 
Nuclide & curies \\
\hline Ba-137m & $2.6000 e+000$ \\
Co-60 & $1.1000 e-002$ \\
Cs-137 & $2.7000 e+000$ \\
Eu-154 & $1.6000 e-001$ \\
Sr-90 & $4.3200 e+001$ \\
y-90 & $4.3200 e+001$
\end{tabular}

Activity

Energy

MeV

photons/sec

0.03

$5.666 e+09$

$2.542 e+09$

$3.060 e+0 B$

$2.395 e+09$

$4.043 e+08$

$4.224 e+07$

$1.282 e+07$

$8.704 e+10$

$2.309 e+09$
Source Input

Grouping Method : Standard Indices

Number of Groups : 25

Lower Energy Cutoff : 0.015

Photons \& 0.015 : Excluded

Library : Grove

$Z$ Direction becquerels

$9.6200 e+010$

$4.0700 e+008$

$9.9900 e+010$

$5.9200 e+009$

$1.5984 e+012$

$1.5984 e+012$

Shield Name

Shield 1

Shield 2

Shield 3

Shield 4

Air Gap

Immersion

\section{$x$}

$72.387 \mathrm{~cm}$

$2 \mathrm{ft} 4.5$ in

Buildup

The material reference is : Shield 3

Integration Parameters

$X$ Direction

Results $\angle \mathrm{Ci} / \mathrm{cm}^{2}$

$8.0317 e+002$

$3.3980 e+000$

B.3407e+002

$4.9426 e+001$

$1.3345 e+004$

$1.3345 e+004$

\begin{tabular}{c}
$\frac{\text { Eluence Rate }}{\text { MeV/cm }}{ }^{2} / \mathrm{sec}$ \\
\hline$\frac{\text { No Buildup }}{1.723 e-21}$ \\
$2.042 e-08$ \\
$2.386 e-04$ \\
$1.428 e+02$ \\
$2.050 e+02$ \\
$6.042 e+01$ \\
$2.459 e+01$ \\
$2.110 e+05$ \\
$8.044 e+03$
\end{tabular}

$8.044 e+03$
0.05

0.2

0.4

0.6
Source Dimensions

$56.896 \mathrm{~cm}$

$56.896 \mathrm{~cm}$

Dose Points<smiles></smiles>
$28.448 \mathrm{~cm}$ 11.2 in

Shields

\begin{tabular}{|c|c|c|}
\hline Dimension & Material & Density \\
\hline $.191 \mathrm{~cm}$ & Iron & 7.86 \\
\hline $10.795 \mathrm{~cm}$ & Air & 0.00122 \\
\hline $.635 \mathrm{~cm}$ & Iron & 7.86 \\
\hline \multirow[t]{3}{*}{$2.87 \mathrm{~cm}$} & Carbon & 0.2 \\
\hline & Air & 0.00122 \\
\hline & Air & 0.00122 \\
\hline
\end{tabular}

$1 \mathrm{ft} 10.4 \mathrm{in}$

$1 \mathrm{ft} 10.4 \mathrm{in}$
$\mathrm{Bg} / \mathrm{cm}^{2}$

$2.9717 e+007$

$1.2573 e+005$

$3.0860 e+007$

$1.8288 e+006$

$4.9377 e+008$

$4.9377 e+008$ 
Page : 2

DOS File: RMDSEXH2.MS5

Run Date: August 3, 2000 RPP-6549

Run Time: 8:08:32 AM

Revision 0

Page 7 of 21

Duration : 00:00:01

$\begin{array}{cc}\frac{\text { Energy }}{\text { MeV }} & \begin{array}{c}\text { Activity } \\ \text { photona/sec }\end{array} \\ 1.0 & 2.228 e+09 \\ 1.5 & 2.717 e+09 \\ \text { TOTALS: } & 1.057 e+11\end{array}$

$\frac{\text { Eluence Rate }}{\text { MeV/em }{ }^{2} / \text { sec }}$
$\frac{\text { No Buildup }}{1.024 e+04}$
$2.039 \mathrm{e}+04$
$2.501 \mathrm{e}+05$

\begin{abstract}
Eluence Rate
$\mathrm{MeV} / \mathrm{cm}^{2} / \mathrm{sec}$

With Buildug

$1.408 \mathrm{e}+04$

$2.588 \mathrm{e}+04$
\end{abstract}

$3.783 e+05$
Exposure Rate

$\mathrm{mR} / \mathrm{hr}$

No Buildup

$1.887 e+01$

$3.431 e+01$

$4.811 e+02$
Exposure Rate $\mathrm{mR} / \mathrm{hr}$

With Buildup

$2.595 e+01$

$4.354 e+01$

$7.291 e+02$ 


\section{Page : 1}

DOS File: RMDSEXH3.MS5

Run Date: June 14, 2000

Run Time: 8:05:24 AM

Duration : 00:00:01
River Protection Project - Radiological Control

File Ref:

Date: $8 / 2 / 2000$

By:

Checked:
Case Title: RCMSExh3

Description: RCMS Exhauster - Case 3

Geometry: 5 - Rectangular Area - Horizontal

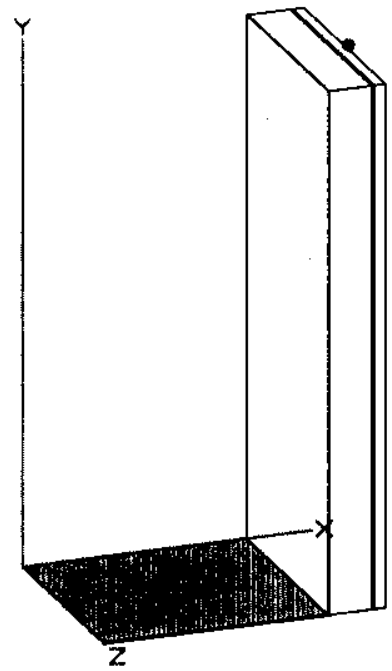

Nuclide

Ba-137m

Co-60

Cs-137

Eu-154

$\mathrm{Sr}-90$

y-90 curies

$2.6000 e+000$

$1.1000 e-002$

$2.7000 e+000$

$1.6000 e-001$

$4.3200 e+001$

$4.3200 e+001$

Source Input

Grouping Method : Standard Indices

Number of Groups : 25

Lower Energy Cutoff : 0.015

Photons < 0.015 : Excluded

Library : Grove

$\begin{array}{cc}\frac{\text { becquerels }}{2.6200 e+010} & \frac{\mathrm{Ci} / \mathrm{cm}^{2}}{8.0317 e+002} \\ 9.0700 e+008 & 3.3980 e+000 \\ 9.9900 e+010 & 8.3407 e+002 \\ 5.9200 e+009 & 4.9426 e+001 \\ 1.5984 e+012 & 1.3345 e+004 \\ 1.5984 e+012 & 1.3345 e+004\end{array}$

Buildup

The material reference is : Shield 3

Integration Parameters

Z Direction

$X$ Direction

Activity

Energy

MeV

0.03

0.04

0.05

0.1

0.2

0.4

photons/sec

$5.666 e+09$

$2.542 e+09$

$3.060 e+08$

$2.395 e+09$

$4.043 e+08$

$4.224 e+07$
Fluence Rate

$\mathrm{MeV} / \mathrm{cm}^{2} / \mathrm{sec}$

No Buildup

$1.035 e-31$

$1.717 e-13$

$1.840 e-07$

$1.351 e+01$

4.291e+01

$1.539 e+01$
Source Dimensions

$56.896 \mathrm{~cm}$

$56.896 \mathrm{~cm}$

Dose Points

$y$

$133.35 \mathrm{~cm}$

$4 \mathrm{ft} 4.5 \mathrm{in}$

$1 \mathrm{ft} 10.4 \mathrm{in}$

$1 \mathrm{ft} 10.4 \mathrm{in}$

Shield 1

Shield 2

Shield 3

Air Gap

Immersion

$2.387 \mathrm{~cm}$

$2 \mathrm{ft} 4.5 \mathrm{in}$

Shields

Dimension

.075 in

4.25 in

$.25 \mathrm{in}$

1.13 in

Material
Iron
Air
Iron
Carbon
Air
Air

Density

7.86

0.00122

7.86

0.2

0.00122

0.00122
$\frac{\mathrm{Bq} / \mathrm{cm}^{2}}{2.9717 e+007}$
$1.2573 e+005$
$3.0860 e+007$
$1.8288 e+006$
$4.9377 e+008$
$4.9377 e+008$ 
Page :2.2

DÓS File: RMDSEXH3.MS5

Run Date: June 14, 2000

Duration : 00:00:01

RPP-6549
Run Time: 8:05:24 AM

Revision 0

Page 9 of 21

$\begin{array}{cccc}\frac{\text { Energy }}{\mathrm{MeV}} & \begin{array}{c}\text { Activity } \\ \text { photons } / \mathrm{sec}\end{array} & \begin{array}{c}\text { Fluence Rate } \\ \frac{\mathrm{MeV} / \mathrm{cm}^{2} / \mathrm{sec}}{\text { No Buildup }}\end{array} & \frac{\text { Fluence Rate }}{\mathrm{MeV} / \mathrm{cm}^{2} / \mathrm{sec}} \\ 0.5 & 1.282 e+07 & 6.526 e+00 & \frac{\text { With Buildup }}{1.311 e+01} \\ 0.6 & 8.704 e+10 & 5.771 e+04 & 1.092 e+05 \\ 0.8 & 2.309 e+09 & 2.298 e+03 & 3.954 e+03 \\ 1.0 & 2.228 e+09 & 3.016 e+03 & 4.849 e+03 \\ 1.5 & 2.717 e+09 & 6.317 e+03 & 9.119 e+03 \\ \text { TOTALS: } & 1.057 e+11 & & 1.273 e+05\end{array}$

$\frac{\text { Exposure Rate }}{\mathrm{mR} / \mathrm{hr}}$
$\frac{\text { No Buildup }}{1.281 e-02}$
$1.126 e+02$
$4.370 e+00$
$5.560 e+00$
$1.063 e+01$

$1.333 e+02$
Exposure Rate $\mathrm{mR} / \mathrm{hr}$

With Buildup

$2.573 e-02$

$2.132 e+02$

$7.521 e+00$

$8.939 e+00$

$1.534 e+01$

$2.453 e+02$ 
Page : 1

DOS File: RMDSEXH4.MS5

Run Date: June 14, 2000

Run Time: 8:22:34 AM

Duration : 00:00:01
Page 10 of 21

File Ref:

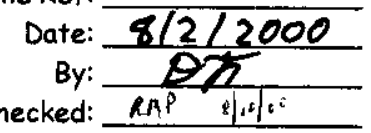

Case Title: RCMSExh4

Description: RCMS Exhauster - Case 4

Geometry: 5 - Rectangular Area - Horizontal

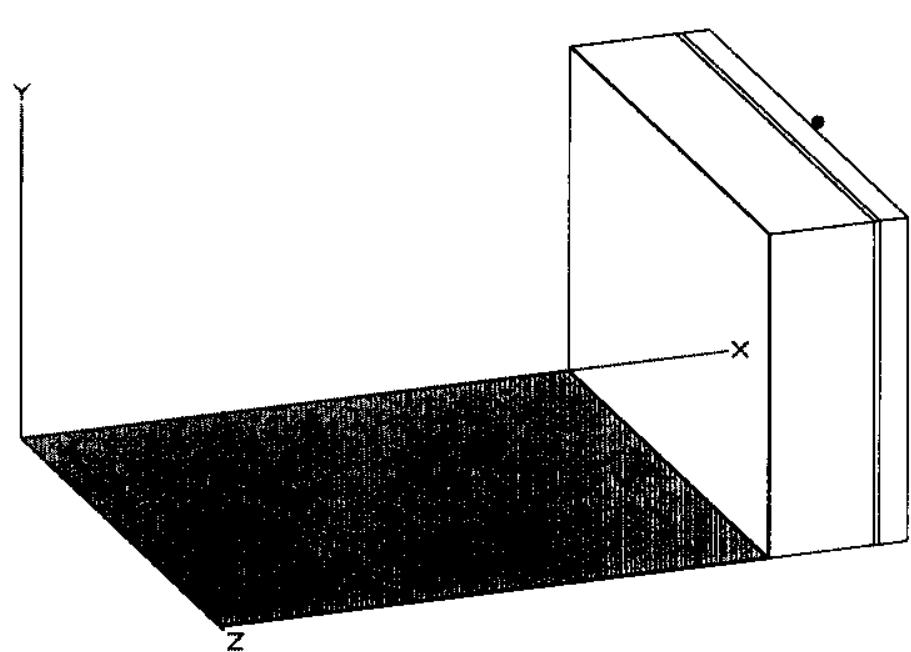

Source Input

Grouping Method : Standard Indices

Number of Groups : 25

Lower Energy Cutoff : 0.015

Photons 0.015 : Excluded

Library : Grove

$\begin{array}{lc}\text { Nuclide } & \text { curies } \\ \text { Ba-137m } & 2.6000 e+000 \\ \text { Co-60 } & 1.1000 e-002 \\ \text { Cs }-137 & 2.7000 e+000 \\ \text { Eu-154 } & 1.6000 e-001 \\ \text { Sr-90 } & 4.3200 e+001 \\ \text { y-90 } & 4.3200 e+001\end{array}$

Nuclide

$\mathrm{Ba}-137 \mathrm{~m}$

becquerels

$9.6200 e+010$

$4.0700 e+008$

$9.9900 e+010$

$5.9200 e+009$

$1.5984 e+012$

Length

Width

\section{Shield Name}

Shield 1

Shield 2

Shield 3

Shield 4

Air Gap

Immersion

$1.5984 e+012$
Source Dimensions

$56.896 \mathrm{~cm}$

$56.896 \mathrm{~cm}$

$\underline{x}$

$72.387 \mathrm{~cm}$

$2 \mathrm{ft} 4.5 \mathrm{in}$

Buildup

The material reference is : Shield 3

Integration Parameters

Z Direction

$X$ Direction

$\frac{\mathrm{Ci} / \mathrm{cm}^{2}}{8.0317 e+002}$
$3.3980 e+000$
$8.3407 e+002$
$4.9426 e+001$
$1.3345 e+004$
$1.3345 e+004$

$\mathrm{Bq} / \mathrm{cm}^{2}$ $2.9717 e+007$ $1.2573 e+005$

$3.0860 e+007$

$1.8288 e+006$

$4.9377 e+008$

$4.9377 e+008$
Dose Points

$1 \mathrm{ft} 10.4$ in

$1 \mathrm{ft} 10.4 \mathrm{in}$

$$
\begin{gathered}
\stackrel{Z}{28.448 \mathrm{~cm}} \\
11.2 \mathrm{in}
\end{gathered}
$$

\begin{tabular}{ccl}
$\begin{array}{r}\text { Shields } \\
\text { Dimension }\end{array}$ & Material & \\
\cline { 1 - 2 } $.075 \mathrm{in}$ & Density \\
$4.25 \mathrm{in}$ & Air & 7.86 \\
$.25 \mathrm{in}$ & Iron & 0.00122 \\
$1.13 \mathrm{in}$ & Carbon & 0.86 \\
& Air & 0.00122 \\
& Air & 0.00122
\end{tabular}




\section{Page : 2}

DOS File: RMDSEXH4.MS5

Run Date: June 14, 2000

Run Time: 8:22:34 AM

Duration : 00:00:01

$\begin{array}{cc}\frac{\text { Energy }}{\text { MeV }} & \begin{array}{c}\text { Activity } \\ \text { photons } / \text { sec }\end{array} \\ 0.5 & 1.282 e+07 \\ 0.6 & 8.704 e+10 \\ 0.8 & 2.309 e+09 \\ 1.0 & 2.228 e+09 \\ 1.5 & 2.717 e+09\end{array}$

\begin{tabular}{cc}
$\frac{\text { Fluence Rate }}{\mathrm{MeV} / \mathrm{cm}^{2} / \mathrm{sec}}$ & $\begin{array}{c}\text { Fluence Rate } \\
\text { No Buildup }\end{array}$ \\
\hline $2.459 e+01$ & $\frac{\mathrm{MeV} / \mathrm{cm}^{2} / \mathrm{sec}}{\text { With Buildup }}$ \\
$2.110 e+05$ & $3.975 e+01$ \\
$8.044 e+03$ & $3.259 e+05$ \\
$1.024 e+04$ & $1.162 e+04$ \\
$2.039 e+04$ & $1.408 e+04$ \\
$2.501 e+05$ & $2.588 e+04$ \\
& $3.783 e+05$
\end{tabular}

Exposure Rate $\mathrm{mR} / \mathrm{hr}$

No Buildup

4.827e-02

$4.119 e+02$

$1.530 e+01$

$1.887 e+01$

$3.431 e+01$

$4.811 e+02$
Exposure Rate $\mathrm{mR} / \mathrm{hr}$

With Buildup

7.802e-02

6.361e+02

$2.210 e+01$

$2.595 e+01$

$4.354 e+01$

$7.291 e+02$ 
Run Date: June 14, 2000

Run Time: 7:14:01 AM

Duration : 00:00:06
File Ref:

Date: $8 / 2 / 2000$

By:

Checked

y:<smiles>[R]OC1CC2CCCC1CC2</smiles>

Case Title: RMCSExh5

Description: RMCS Exhauster - Case 5

Geometry: 13 - Rectangular Volume

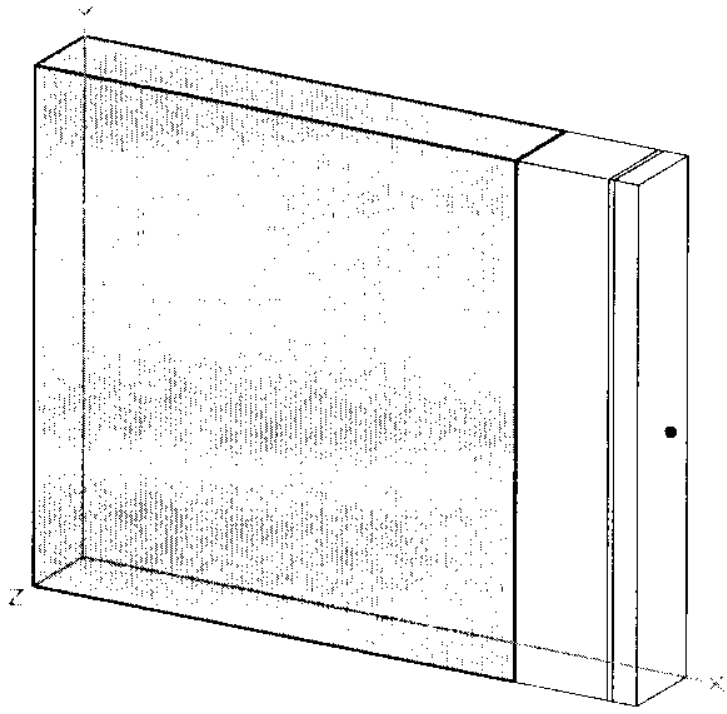

$\begin{array}{lc}\frac{\text { Nuclide }}{\text { Ba-137m }} & \underline{\text { curies }} \\ \text { Co-60 } & 2.6000 e+000 \\ \text { Cs-137 } & 1.1000 e-002 \\ \text { Eu-154 } & 2.7000 e+000 \\ \text { Sr-90 } & 1.6000 e-001 \\ \text { Y-90 } & 4.3200 e+001 \\ & 4.3200 e+001\end{array}$

Source Dimensions

$56.896 \mathrm{~cm}$

$10.16 \mathrm{~cm}$

$56.896 \mathrm{~cm}$

Dose Points

$$
\begin{array}{r}
\frac{y}{28.448 \mathrm{~cm}} \\
11.2 \mathrm{in}
\end{array}
$$

$1 \mathrm{ft} 10.4 \mathrm{in}$ $4.0 \mathrm{in}$

$1 \mathrm{ft} 10.4 \mathrm{in}$
Source Input

Grouping Method : Standard Indices

Number of Groups : 25

Lower Energy Cutoff : 0.015

Photons \& 0.015 : Excluded

Library : Grove becquerels

$9.6200 e+010$

$4.0700 e+008$

$9.9900 e+010$

$5.9200 e+009$

$1.5984 e+012$

$1.5984 e+012$

Shield Name
Source
Shield 1
Shield 2
Shield 3
Shield 4
Air Gop

Shield Name

Shield 1

Shield 2

Shield

$72.39 \mathrm{~cm}$

$2 \mathrm{ft} 4.5 \mathrm{in}$

Buildup

The material reference is : Shield 3

Integration Parameters

$x$ Direction

$y$ Direction

$Z$ Direction

$\begin{array}{cc}\frac{\mathrm{Ki} / \mathrm{cm}^{3}}{7.9053 e+001} & \frac{\mathrm{Bg} / \mathrm{cm}^{3}}{2.9249 e+006} \\ 3.3445 e-001 & 1.2375 e+004 \\ 8.2093 e+001 & 3.0374 e+006 \\ 4.8648 e+000 & 1.8000 e+005 \\ 1.3135 e+003 & 4.8599 e+007 \\ 1.3135 e+003 & 4.8599 e+007\end{array}$

$\frac{Z}{Z} .08 \mathrm{~cm}$

\begin{tabular}{|c|c|c|}
\hline Dimenșion & Material & Density \\
\hline $2007.04 \mathrm{in}^{3}$ & HEPA & 0.05 \\
\hline $.075 \mathrm{in}$ & Iron & 7.86 \\
\hline $4.25 \mathrm{in}$ & Air & 0.00122 \\
\hline $25 \mathrm{in}$ & Iron & 7.86 \\
\hline \multirow[t]{2}{*}{$1.13 \mathrm{in}$} & Carbon & 0.29 \\
\hline & Air & 0.00122 \\
\hline
\end{tabular}

$2.0 \mathrm{in}$

Shields

$\begin{array}{ccc}\frac{\text { Energy }}{\text { MeV }} & \begin{array}{c}\text { Activity } \\ \text { photons/sec }\end{array} & \frac{\text { Fluence Rate }}{\text { MeV/cm } / \mathrm{sec}} \\ 0.03 & 5.666 e+09 & \frac{\text { No Buildup }}{2.588 e-20} \\ 0.04 & 2.542 e+09 & 1.413 e-07 \\ 005 & 3.060 e+08 & 1.322 e-03 \\ 01 & 2.395 e+09 & 8.029 e+02\end{array}$

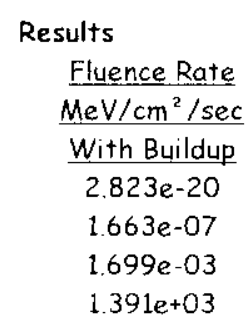

10

20

20 
Page :2

DOS File: RMDSEXH5.MS5

Run Date: June 14, 2000

Run Time: 7:14:01 AM

RPP-6549

Duration : 00:00:06

Revision 0

Page 13 of 21

\begin{tabular}{|c|c|c|c|c|c|}
\hline \multirow{3}{*}{$\mathrm{MeV}$} & Activity & Fluence Rate & Fluence Rate & Exposure Rate & Exposure Rate \\
\hline & photons/sec & $\mathrm{MeV} / \mathrm{cm}^{2} / \mathrm{sec}$ & $\mathrm{MeV} / \mathrm{cm}^{2} / \mathrm{sec}$ & $\mathrm{mR} / \mathrm{hr}$ & $\mathrm{mR} / \mathrm{hr}$ \\
\hline & & No Buildup & With Buildup & No Buildup & With Buildup \\
\hline 0.2 & $4.043 e+08$ & $1.249 e+03$ & $2.542 e+03$ & $2.205 e+00$ & $4.486 e+00$ \\
\hline 0.4 & $4.224 e+07$ & $3.846 e+02$ & $7.097 e+02$ & $7.494 e-01$ & $1.383 e+00$ \\
\hline 0.5 & $1.282 e+07$ & $1.584 e+02$ & $2.756 e+02$ & $3.110 e-01$ & $5.410 e-01$ \\
\hline 0.6 & $8.704 e+10$ & $1.372 e+06$ & $2.270 e+06$ & $2.678 e+03$ & $4.431 e+03$ \\
\hline 0.8 & $2.309 e+09$ & $5,303 e+04$ & $8.125 e+04$ & $1.009 e+02$ & $1.546 e+02$ \\
\hline 1.0 & $2.228 e+09$ & $6.817 e+04$ & $9.904 e+04$ & $1.257 e+02$ & $1.826 e+02$ \\
\hline 1.5 & $2.717 e+09$ & $1.381 e+05$ & $1.835 e+05$ & $2.323 e+02$ & $3.088 e+02$ \\
\hline TOTALS: & $1.057 e+11$ & $1.634 e+06$ & $2.639 e+06$ & $3.142 e+03$ & $5.085 e+03$ \\
\hline
\end{tabular}


Page : 1

DOS File: RMDSEXH6.MS5

Run Date: June 14, 2000

Run Time: 8:27:45 AM

Duration : 00:00:01
Page 14 of 21
File Ref:

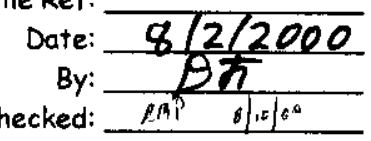

Case Title: RCMSExh6

Description: RCMS Exhauster - Case 6

Geometry: 5 - Rectangular Area - Horizontal

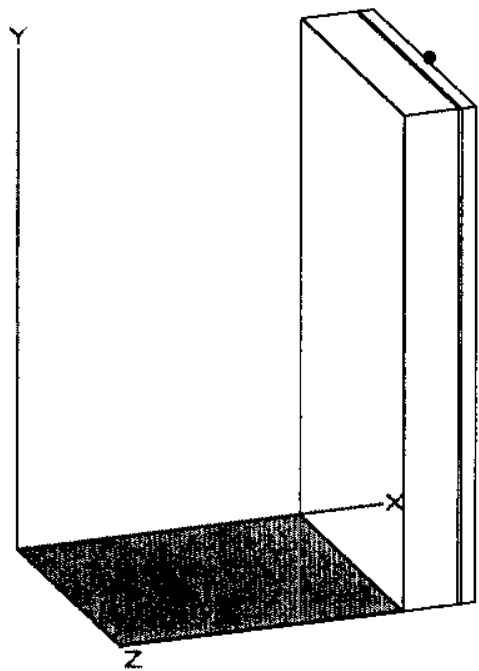

Ba-137m

Co-60

Cs-137

Eu-154

$\mathrm{Sr}-90$

y-90 curies

$2.6000 e+000$

$1.1000 e-002$

$2.7000 e+000$

$1.6000 e-001$

$4.3200 e+001$

$4.3200 e+001$
Z Direction

$X$ Direction
Source Dimensions

$56.896 \mathrm{~cm}$

$56.896 \mathrm{~cm}$

Width

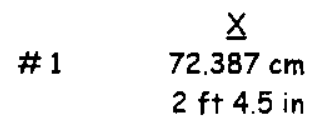

Shield Name

Shield 1

Shield 2

Shield 3

Shield 4

Air Gap

Immersion

Source Input

Grouping Method : Standard Indices

Number of Groups : 25

Lower Energy Cutoff : 0.015

Photons 0.015 : Excluded

Library : Grove

$\begin{array}{cc}\text { becquerels } & \frac{\mathrm{Li} / \mathrm{cm}^{2}}{8.0317 e+002} \\ 9.6200 e+010 & 3.3980 e+000 \\ 4.0700 e+008 & 8.3407 e+002 \\ 9.9900 e+010 & 4.9426 e+001 \\ 5.9200 e+009 & 1.3345 e+004 \\ 1.5984 e+012 & 1.3345 e+004 \\ 1.5984 e+012 & \end{array}$

Buildup

The material reference is : Shield 3

Integration Parameters

Shields $99.695 \mathrm{~cm}$ $3 \mathrm{ft} 3.3 \mathrm{in}$
$1 \mathrm{ft} 10.4 \mathrm{in}$

$1 \mathrm{ft} 10.4$ in

\begin{tabular}{|c|c|c|}
\hline Dimension & Material & Density \\
\hline .075 in & Iron & 7.86 \\
\hline $4.25 \mathrm{in}$ & Air & 0.00122 \\
\hline $.25 \mathrm{in}$ & Iron & 7.86 \\
\hline \multirow[t]{3}{*}{$1.13 \mathrm{in}$} & Carbon & 0.2 \\
\hline & Air & 0.00122 \\
\hline & Air & 0.00122 \\
\hline
\end{tabular}

\section{$\underline{Z}$ $28.448 \mathrm{~cm}$ 11.2 in Density
7.86
0.00122
7.86
0.2
0.00122
0.00122}

$\mathrm{Bq} / \mathrm{cm}^{2}$ $2.9717 e+007$ $1.2573 e+005$ $3.0860 e+007$ $1.8288 e+006$ $4.9377 e+008$ $4.9377 e+008$

$\begin{array}{cc}\frac{\text { Energy }}{\text { MeV }} & \begin{array}{c}\text { Activity } \\ \text { photons } / \text { sec }\end{array} \\ 0.03 & 5.666 e+09 \\ 0.04 & 2.542 e+09 \\ 0.05 & 3.060 e+08 \\ 0.1 & 2.395 e+09 \\ 0.2 & 4.043 e+08 \\ 0.4 & 4.224 e+07\end{array}$

Results

$\frac{\text { Fluence Rate }}{\text { MeV/cm } / \mathrm{cm}^{2} / \mathrm{sec}}$
$\frac{\text { With Buildup }}{6.419 e-24}$
$2.043 e-11$
$3.799 e-06$
$6.066 e+01$
$1.674 e+02$
$5.015 e+01$


Page : 2

DOS File: RMDSEXH6.MS5

Run Date: June 14, 2000

Run Time: 8:27:45 AM

RPP-6549

Revision 0

Page 15 of 21

Duration: 00:00:01

$\begin{array}{ccc}\begin{array}{c}\text { Energy } \\ \text { MeV }\end{array} & \begin{array}{c}\text { Activity } \\ \text { photons/sec }\end{array} & \begin{array}{c}\begin{array}{c}\text { Fluence Rate } \\ \text { MeV/cm } / \mathrm{sec}\end{array} \\ \text { No Buildup }\end{array} \\ 0.5 & 1.282 e+07 & 1.063 e+01 \\ 0.6 & 8.704 e+10 & 9.286 e+04 \\ 0.8 & 2.309 e+09 & 3.633 e+03 \\ 1.0 & 2.228 e+09 & 4.710 e+03 \\ 1.5 & 2.717 e+09 & 9.666 e+03 \\ \text { TOTALS: } & 1.057 e+11 & 1.110 e+05\end{array}$

$\frac{\text { Fluence Rate }}{\text { MeV/cm } / \mathrm{sec}}$
$\frac{\text { With Buildup }}{1.963 e+01}$
$1.623 e+05$
$5.831 e+03$
$7.125 e+03$
$1.330 e+04$
$1.888 e+05$

Exposure Rate

$\mathrm{mR} / \mathrm{hr}$

No Buildup

2.086e-02

$1.813 e+02$

$6.910 e+00$

$8.681 e+00$

$1.626 e+01$

Exposure Rate $\mathrm{mR} / \mathrm{hr}$

With Buildup

3.852e-02

$3.168 e+02$

$1.109 e+01$

$1.313 e+01$

$2.238 e+01$

$2.134 e+02$

$3.639 e+02$ 


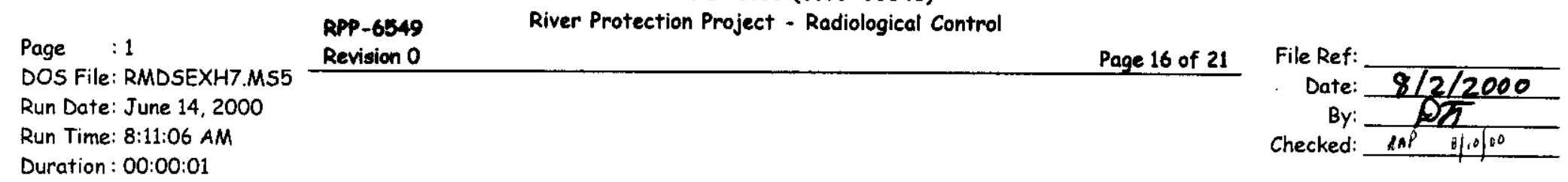

Case Title: RCMSExh7

Description: RCMS Exhauster - Case 7

Geometry: 5 - Rectangular Area - Horizontal
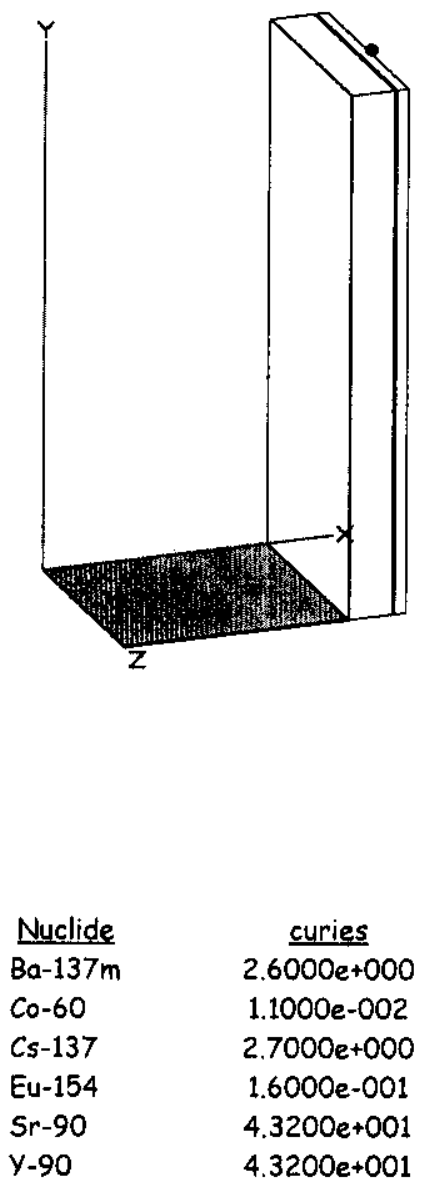

Source Input

Grouping Method : Standard Indices

Number of Groups : 25

Lower Energy Cutoff : 0.015

Photons \& 0.015 : Excluded

Library: Grove

\begin{tabular}{|c|c|c|}
\hline becquerels & $\mathrm{Li} / \mathrm{cm}^{2}$ & $\mathrm{~Bq} / \mathrm{cm}^{2}$ \\
\hline $9.6200 e+010$ & $8.0317 e+002$ & $2.9717 e+007$ \\
\hline $4.0700 e+008$ & $3.3980 \mathrm{e}+000$ & $1.2573 e+005$ \\
\hline $9.9900 e+010$ & $8.3407 e+002$ & $3.0860 e+007$ \\
\hline $5.9200 e+009$ & $4.9426 e+001$ & $1.8288 e+006$ \\
\hline $1.5984 e+012$ & $1.3345 e+004$ & $4.9377 e+008$ \\
\hline $1.5984 e+012$ & $1.3345 e+004$ & $4.9377 e+008$ \\
\hline
\end{tabular}

Buildup

The material reference is : Shield 3

Integration Parameters

Z Direction

$X$ Direction

Activity

\begin{tabular}{c}
$\frac{\text { Fluence Rate }}{\text { MeV/cm }}{ }^{2} / \mathrm{sec}$ \\
\hline$\frac{\text { No Buildup }}{1.035 e-31}$ \\
$1.717 e-13$ \\
$1.840 e-07$ \\
$1.351 e+01$ \\
$4.291 e+01$ \\
$1.539 e+01$
\end{tabular}

Results

$$
\begin{gathered}
\frac{\text { Fluence Rate }}{\text { MeV/cm }}{ }^{2} / \mathrm{sec} \\
\hline \text { With Buildup } \\
\hline 4.594 e-24 \\
2.056 e-13 \\
2.431 e-07 \\
2.584 e+01 \\
1.008 e+02 \\
3.307 e+01
\end{gathered}
$$

20

20
Source Dimensions

$56.896 \mathrm{~cm}$

$56.896 \mathrm{~cm}$

\begin{tabular}{|c|c|c|}
\hline Dimension & Material & Density \\
\hline .075 in & Iron & 7.86 \\
\hline 4.25 in & Air & 0.00122 \\
\hline $.25 \mathrm{in}$ & Iron & 7.86 \\
\hline \multirow[t]{3}{*}{$1.13 \mathrm{in}$} & Carbon & 0.2 \\
\hline & Air & 0.00122 \\
\hline & Air & 0.00122 \\
\hline
\end{tabular}

$1 \mathrm{ft} 10.4$ in

$1 \mathrm{ft} 10.4 \mathrm{in}$

Dose Points

$$
\begin{aligned}
& 133.35 \mathrm{~cm} \\
& 4 \mathrm{ft} 4.5 \mathrm{in}
\end{aligned}
$$

Z

$28.448 \mathrm{~cm}$

11.2 in

Shields photons/sec

$5.666 e+09$

$2.542 e+09$

$3.060 e+08$

$2.395 e+09$

$4.043 e+08$

$4.224 e+07$

$\begin{array}{cc}\frac{\text { Energy }}{\text { MeV }} & \begin{array}{c}\text { Activity } \\ \text { photons/sec }\end{array} \\ 0.03 & 5.666 e+09 \\ 0.04 & 2.542 e+09 \\ 0.05 & 3.060 e+08 \\ 0.1 & 2.395 e+09 \\ 0.2 & 4.043 e+08 \\ 0.4 & 4.224 e+07\end{array}$

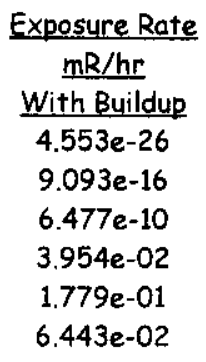


Page : ?

DOS File: RMDSEXH7.MS5

Run Date: June 14, 2000

Run Time: 8:11:06 AM

Duration : 00:00:01

\begin{tabular}{|c|c|c|c|c|c|}
\hline Eneray & Activity & Eluence Rate & Fluence Rate & Exposure Rate & Exposure Rate \\
\hline \multirow[t]{2}{*}{ MeV } & photons/sec & $\mathrm{MeV} / \mathrm{cm}^{2} / \mathrm{sec}$ & $\mathrm{MeV} / \mathrm{cm}^{2} / \mathrm{sec}$ & $\underline{\mathrm{mR} / \mathrm{hr}}$ & $\mathrm{mR} / \mathrm{hr}$ \\
\hline & & No Buildup & With Buildup & No Buildup & With Buildup \\
\hline 0.5 & $1.282 e+07$ & $6.526 e+00$ & $1.311 e+01$ & $1.281 e-02$ & $2.573 e-02$ \\
\hline 0.6 & $8.704 e+10$ & $5.771 e+04$ & $1.092 e+05$ & $1.126 e+02$ & $2.132 e+02$ \\
\hline 0.8 & $2.309 e+09$ & $2.298 e+03$ & $3.954 e+03$ & $4.370 e+00$ & $7.521 e+00$ \\
\hline 1.0 & $2.228 e+09$ & $3.016 e+03$ & $4.849 e+03$ & $5.560 e+00$ & $8.939 e+00$ \\
\hline 1.5 & $2.717 e+09$ & $6.317 e+03$ & $9.119 e+03$ & $1.063 e+01$ & $1.534 e+01$ \\
\hline TOTALS: & $1.057 e+11$ & $6.942 e+04$ & $1.273 e+05$ & $1.333 e+02$ & $2.453 e+02$ \\
\hline
\end{tabular}


Page : 1

DOS File: RMDSEXH8.MS5

Run Date: June 14, 2000

Run Time: 8:30:5? AM

Duration : 00:00:01

File Ref:

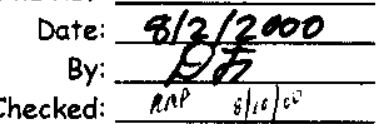

Case Title: RCMSExh8

Description: RCMS Exhauster - Case 8

Geometry: 5 - Rectangular Area - Horizontal

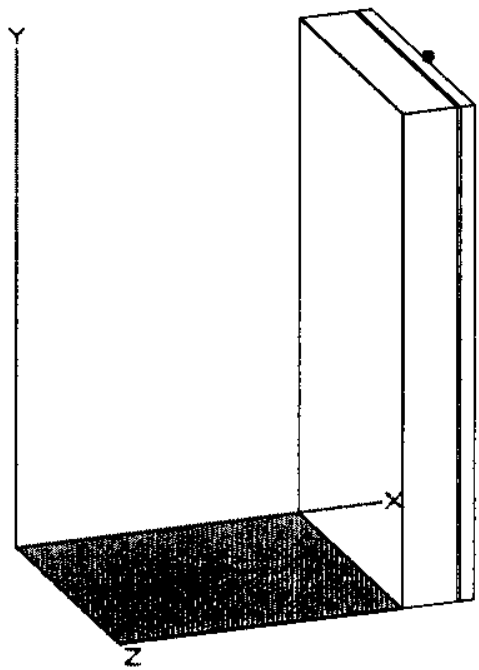

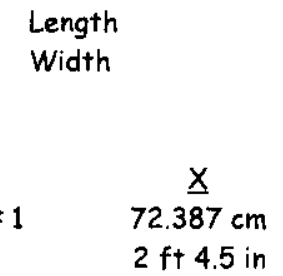

Source Dimensions

$56.896 \mathrm{~cm}$

$1 \mathrm{ft} 10.4 \mathrm{in}$

$56.896 \mathrm{~cm}$

$1 \mathrm{ft} 10.4 \mathrm{in}$

\section{Dose Points

$$
\begin{aligned}
& 99.695 \mathrm{~cm} \\
& 3 \mathrm{ft} 3.3 \mathrm{in}
\end{aligned}
$$

Z

$28.448 \mathrm{~cm}$

11.2 in

Shields

\begin{tabular}{l} 
Shield Name \\
\hline Shield 1 \\
Shield 2 \\
Shield 3 \\
Shield 4 \\
Air Gap \\
Immersion
\end{tabular}

Source Input

Grouping Method : Standard Indices

Number of Groups : 25

Lower Energy Cutoff : 0.015

Photons < 0.015 : Excluded

\begin{tabular}{|c|c|}
\hline Nuclide & curies \\
\hline $\mathrm{Ba}-137 \mathrm{~m}$ & $2.6000 e+000$ \\
\hline Co-60 & $1.1000 \mathrm{e}-002$ \\
\hline Cs-137 & $2.7000 e+000$ \\
\hline Eu-154 & $1.6000 e-001$ \\
\hline $5 r-90$ & $4.3200 e+001$ \\
\hline$y-90$ & $4.3200 e+001$ \\
\hline
\end{tabular}

Library : Grove

becquerels

$9.6200 e+010$

$1 \mathrm{Ci} / \mathrm{cm}^{2}$

$4.0700 e+008$

$8.0317 e+002$

$3.3980 e+000$

$9.9900 e+010$

$8.3407 e+002$

$5.9200 e+009$

$4.9426 e+001$

$1.5984 e+012$

$1.3345 e+004$

$1.5984 e+012$

\begin{tabular}{|c|c|c|}
\hline \multicolumn{3}{|c|}{ Shields } \\
\hline Dimension & Material & Density \\
\hline .075 in & Iron & 7.86 \\
\hline $4.25 \mathrm{in}$ & Air & 0.00122 \\
\hline $.25 \mathrm{in}$ & Iron & 7.86 \\
\hline 1.13 in & Carbon & 0.2 \\
\hline & Air & 0.00122 \\
\hline & Air & 0.00122 \\
\hline
\end{tabular}

$1.3345 e+004$

\section{Buildup}

The material reference is : Shield 3

Integration Parameters

Z Direction

$X$ Direction
20

\begin{tabular}{|c|c|}
\hline Energy & Activity \\
\hline $\mathrm{MeV}$ & photons/sec \\
\hline 0.03 & $5.666 e+09$ \\
\hline 0.04 & $2.542 e+09$ \\
\hline 0.05 & $3.060 e+08$ \\
\hline 0.1 & $2.395 e+09$ \\
\hline 0.2 & $4.043 e+08$ \\
\hline 0.4 & $4.224 e+07$ \\
\hline
\end{tabular}

20

\begin{tabular}{c}
$\frac{\text { Fluence Rate }}{\text { MeV/cm }} \mathrm{cm}^{2} / \mathrm{sec}$ \\
\hline No Buildup \\
\hline $1.580 e-27$ \\
$1.717 e-11$ \\
$2.903 e-06$ \\
$3.311 e+01$ \\
$7.686 e+01$ \\
$2.549 e+01$
\end{tabular}

Results

$\frac{\text { Fluence Rate }}{\text { MeV/cm } / \mathrm{cm}^{2} / \mathrm{sec}}$
$\frac{\text { With Buildup }}{6.419 e-24}$
$2.043 e-11$
$3.799 e-06$
$6.066 e+01$
$1.674 e+02$
$5.015 e+01$

$5.015 e+01$
$\mathrm{Bq} / \mathrm{cm}^{2}$

$2.9717 e+007$

$1.2573 e+005$

$3.0860 e+007$

$1.8288 e+006$

$4.9377 e+008$

$4.9377 e+008$

0.4

$4.224 e+07$

Exposure Rate $\mathrm{mR} / \mathrm{hr}$

With Buildup

6.361e-26

$9.034 e-14$

$1.012 e-08$

$9.280 \mathrm{e}-02$

2.954e-01

$9.772 e-02$ 
Page : 2

DOS File: RM̉DSEXH8.MS5

Run Date: June 14, 2000

Run Time: 8:30:58 AM

Duration : 00:00:01

RPP-6549

Revision 0

Page 19 of 21

\begin{tabular}{|c|c|c|c|c|c|}
\hline$\frac{\text { Energy }}{\text { MeV }}$ & $\frac{\text { Activity }}{\text { photons/sec }}$ & $\begin{array}{l}\text { Eluence Rate } \\
\mathrm{MeV} / \mathrm{cm}^{2} / \mathrm{sec}\end{array}$ & $\begin{array}{l}\text { Fluence Rate } \\
\text { MeV/cm } / \mathrm{cm}^{2} / \mathrm{sec}\end{array}$ & $\frac{\text { Exposure Rate }}{m R / h r}$ & $\frac{\text { Exposure Rate }}{m R / h r}$ \\
\hline & & No Buildup & With Buildup & No Buildup & With Buildup \\
\hline 0.5 & $1.282 e+07$ & $1.063 e+01$ & $1.963 e+01$ & $2.086 e-02$ & $3.852 e-02$ \\
\hline 0.6 & $8.704 e+10$ & $9.286 e+04$ & $1.623 e+05$ & $1.813 e+02$ & $3.168 e+02$ \\
\hline 0.8 & $2.309 e+09$ & $3.633 e+03$ & $5.831 e+03$ & $6.910 e+00$ & $1.109 e+01$ \\
\hline 1.0 & $2.228 e+09$ & $4.710 e+03$ & $7.125 e+03$ & $8.681 e+00$ & $1.313 e+01$ \\
\hline 1.5 & $2.717 e+09$ & $9.666 e+03$ & $1.330 e+04$ & $1.626 e+01$ & $2.238 e+01$ \\
\hline TOTALS: & $1.057 e+11$ & $1.110 e+05$ & $1.888 e+05$ & $2.134 e+02$ & $3.639 e+02$ \\
\hline
\end{tabular}


Page : 1

DOS File: RMDSEXH9.MS5

RPP -6549

Revision 0

Run Date: June 14, 2000

Run Time: 7:30:38 AM

Duration: 00:00:07
Page 20 of 21

File Ref:

Date: $8 / 2 / 2000$

By:

Checked:

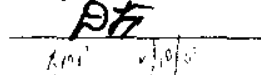

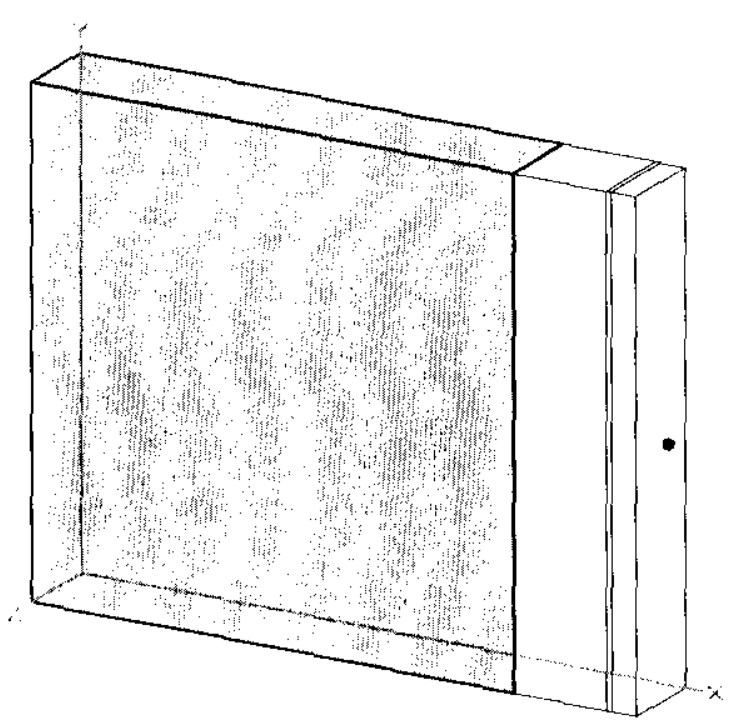

Case Title: RMCSExh9

Description: RMCS Exhauster - Case 9

Geometry: 13 - Rectangular Volume

Length

Width

Height

Shield Name

Source

Shield 1

Shieid 2

Shield 3

Shield 4

Air Gap
Source Input

Grouping Method : Standard Indices

Number of Groups : 25

Lower Energy Cutoff : 0.015

Photons \& 0.015 : Excluded

Library : Grove

$\begin{array}{lc}\text { Nuclide } & \text { curies } \\ \text { Ba-137m } & 2.6000 e+000 \\ \text { Co-60 } & 1.1000 e-002 \\ \text { Cs-137 } & 2.7000 e+000 \\ \text { Eu-154 } & 1.6000 e-001 \\ \text { Sr-90 } & 4.3200 e+001 \\ \text { y-90 } & 4.3200 e+001\end{array}$

becquereis

$9.6200 e+010$

$4.0700 e+008$

$9.9900 e+010$

$5.9200 e+009$
$\mathrm{HCi} / \mathrm{cm}^{3}$

$7.9053 e+001$

3.3445e-001

$8.2093 e+001$

$4.8648 e+000$

$1.3135 e+003$

$1.3135 e+003$
$1 \mathrm{ft} 10.4 \mathrm{in}$

4.0 in

$1 \mathrm{ft} 10.4 \mathrm{in}$

$\begin{array}{lr}\text { Source Dimensions } & \\ 56.896 \mathrm{~cm} & 1 \mathrm{ft} 10.4 \mathrm{in} \\ 10.16 \mathrm{~cm} & 4.0 \mathrm{in} \\ 56.896 \mathrm{~cm} & 1 \mathrm{ft} 10.4 \mathrm{in}\end{array}$

Dose Points

$\begin{array}{rr}\underline{y} & \underline{Z} \\ 28.448 \mathrm{~cm} & 5.08 \mathrm{~cm} \\ 11.2 \mathrm{in} & 2.0 \mathrm{in}\end{array}$

Shields

\begin{tabular}{|c|c|c|}
\hline \multicolumn{3}{|c|}{ Shields } \\
\hline Dimension & Material & Density \\
\hline $2007.04 \mathrm{in}^{3}$ & HEPA & 0.05 \\
\hline .075 in & Iron & 7.86 \\
\hline 4.25 in & Air & 0.00122 \\
\hline $.25 \mathrm{in}$ & Iron & 7.86 \\
\hline $1.13 \mathrm{in}$ & Carbon & 0.29 \\
\hline & Air & 0.00122 \\
\hline
\end{tabular}

$8 \mathrm{~cm}$
$\underline{B} q / \mathrm{cm}^{3}$

$2.9249 e+006$

$1.2375 e+004$

$3.0374 e+006$

$1.8000 e+005$

$4.8599 e+007$

$4.8599 e+007$ $\times$ Direction

Buildup

The material reference is : Shield 3

Integration Parameters

\section{$Y$ Direction}

Z Direction
10

20

20

$\begin{array}{ccc}\begin{array}{c}\text { Energy } \\ \text { MeV }\end{array} & \begin{array}{c}\text { Activity } \\ \text { photons/sec }\end{array} & \frac{\text { Fluence Rate }}{\text { MeV/cm } / \mathrm{sec}} \\ 0.03 & 5.666 e+09 & \frac{\text { No Buildup }}{2.588 e-20} \\ 0.04 & 2.542 e+09 & 1.413 e-07 \\ 0.05 & 3.060 e+08 & 1.322 e-03 \\ 0.1 & 2.395 e+09 & 8.029 e+02\end{array}$

Results

$\frac{\text { Fluence Rate }}{\mathrm{MeV} / \mathrm{cm}^{2} / \mathrm{sec}}$
$\frac{\text { With Buildup }}{2.823 e-20}$
$1.663 e-07$
$1.699 e-03$
$1.391 \mathrm{e}+03$

$\frac{\text { Exposure Rate }}{m R / h r}$
$\frac{m R / h \text { Bildup }}{\text { No But }}$
$2.565 e-22$
$6.247 e-10$
$3.522 e-06$
$1.228 e+00$

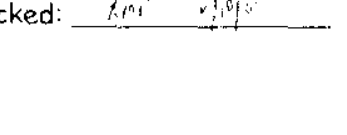


Page : 2

DOS File: RMDSEXH9.MS5

Run Date: June 14, 2000

Run Time: 7:30:38 AM

Duration: 00:00:07

\begin{tabular}{|c|c|c|c|c|c|}
\hline$\frac{\text { Energy }}{\text { MeV }}$ & $\begin{array}{c}\text { Activity } \\
\text { photons/sec }\end{array}$ & $\begin{array}{l}\text { Fluence Rate } \\
\mathrm{MeV} / \mathrm{cm}^{2} / \mathrm{sec}\end{array}$ & $\frac{\text { Fluence Rate }}{\mathrm{MeV} / \mathrm{cm}^{2} / \mathrm{sec}}$ & $\begin{array}{c}\text { Exposure Rate } \\
m R / h r\end{array}$ & $\frac{\text { Exposure Rate }}{m R / h r}$ \\
\hline 02 & & No Buildup & With Buildup & No Buildup & With Buildup \\
\hline 0.2 & $4.043 e+08$ & $1.249 e+03$ & $2.542 e+03$ & $2.205 e+00$ & $4.486 e+00$ \\
\hline $\begin{array}{l}0.4 \\
\end{array}$ & $4.224 e+07$ & $3,846 e+02$ & $7.097 e+02$ & $7.494 e-01$ & $1.383 e+00$ \\
\hline 0.5 & $1.282 e+07$ & $1.584 e+02$ & $2.756 e+02$ & $3.110 e-01$ & $5,410 e-01$ \\
\hline 0.6 & $8.704 e+10$ & $1,372 e+06$ & $2.270 e+06$ & $2.678 \mathrm{e}+03$ & $4.431 e+03$ \\
\hline 0.8 & $2.309 p+09$ & $5.303 e+04$ & $8.125 e+04$ & $1.009 e+02$ & $1.546 e+02$ \\
\hline 1.0 & $2.228 e+09$ & $6.817 e+04$ & $9.904 e+04$ & $1.257 e+02$ & $1.826 e+02$ \\
\hline 1.5 & $2.717 e+09$ & $1.381 e+05$ & $1.835 e+05$ & $2.323 \mathrm{e}+02$ & $3.088 \mathrm{e}+02$ \\
\hline TOTALS: & $1.057 e+11$ & $1.634 e+06$ & $2.639 e+06$ & $3.142 e+03$ & $5.085 e+03$ \\
\hline
\end{tabular}

